\title{
In vitro biochemical features in calli derived from winter wheat anthers and their possible influences on a secondary embryogenesis
}

\author{
Lyubushkina I. ${ }^{1,2 *}$, Polyakova M. ${ }^{1}$, Pomortsev A. ${ }^{1}$, Kirichenko K. ${ }^{1}$, Sokolova N. ${ }^{1}$, \\ Arbuzova G. ${ }^{1,2}$, Voinikov V. ${ }^{1}$, Anapiyaev B. ${ }^{3}$ \\ ${ }^{1}$ Siberian Institute of Plant Physiology and Biochemistry SB RAS, Irkutsk, Russia \\ ${ }^{2}$ Irkutsk State University, Irkutsk, Russia \\ ${ }^{3}$ Satbayev University, Almaty, Kazakhstan \\ * email: ostrov1873@yandex.ru
}

Nowadays a new findings in double haploid technologies (DHT) let to improve their efficiency and applicability in a world breeding programs. However there are a lot of difficulties in practice of DHT, especial in the area of winter crops using. Thus, in winter wheat, along with embryo-like structures in the culture of isolated anthers, a significant number of rhizogenic calli are formed. They are incapable of secondary embryoidogenesis, and this fact negatively affects the final yield of green regenerant plants. In this work, we investigated the changes of energy metabolism and fatty acid composition in the rhizogenic calli, derived from isolated anthers of winter wheat variety Irkutskaya. This will let us reveal possible influence of rhizogenic calli metabolism features preventing the transition to secondary embryogenesis in the culture. It was shown the respiration rate of rhizogenic calli was about $100 \mathrm{nmol} \mathrm{O}_{2} /(\mathrm{min} * \mathrm{~g}$ fresh weight), while the contribution of alternative pathway to respiration was no more than $10 \%$. At the same time, the high respiration rate was provided mainly due to the increased respiratory activity of the roots formed in these calli. The fatty acid composition of rhizogenic calli was distinguished by an increased content of saturated fatty acids, including pentadecanoic and heptadecanoic acids performed regulatory functions, and a low content of unsaturated fatty acids. Thus, we can conclude that the studied features of the rhizogenic calli metabolism indicate an insufficient supply of cells with the energy necessary to maintain the processes of morphogenesis.

Acknowledgements: The reported study was funded by RFBR, project number 20-34-80003. The research was done using the collections of The Core Facilities Center"Bioresource Center" and the equipment of The Core Facilities Center "Bioanalitika" at Siberian Institute of Plant Physiology and Biochemistry SB RAS (Irkutsk, Russia). 